\title{
SENTIMENT ANALYSIS ON GOOGLE CLOUD PLATFORM
}

\author{
Terrence E. White, University of St. Thomas, tewhite@stthomas.edu \\ Manjeet Rege, University of St. Thomas, rege@stthomas.edu
}

\begin{abstract}
This project explores two services available on the Google Cloud Platform (GCP) for performing sentiment analysis: Natural Language API and AutoML Natural Language. The former provides powerful prebuilt models that can be invoked as a service allowing developers to perform sentiment analysis, along with other features like entity analysis, content classification, and syntax analysis. The latter allows developers to train more domain specific models whenever the pre-trained models offered by the Natural Language API are not sufficient. Experiments have been conducted with both of these offerings and the results are presented herein.
\end{abstract}

Keywords: Google Cloud Platform, GCP, Natural Language API, AutoML, Sentiment Analysis

\section{INTRODUCTION}

Given the immense proliferation of online comments and opinions dominating a wide variety of social media and news platforms, automated sentiment analysis has quickly become a necessity in order to quickly identify and purge unwanted messages and message producers from online shared spaces, but they can be very difficult to accurately identify (Islam, 2017). Negative content can quickly lead to disinformation campaigns (Bandeli, 2018), bullying, and other types of toxic dialog that can turn an online platform into a potentially dangerous place for its users (Buzzetto-More, 2015). Finding effective ways to address this issue on a large scale is critical in order to combat the ever-increasing influx of toxic messaging on our social platforms. Data scientist working in this field must also understand the subjective nature of message toxicity, especially as it relates to the difficulties of properly labeling comments in test datasets. In some cases, messages can truly be positive or negative, but if comments are the result of an emerging threat, they can potentially be informative of people's emotional state and best efforts to adapt to a challenging event (Gaspar, 2015). Understanding these and other issues that arise in sentiment analysis is critical to ensuring the accuracy of the results (Brown, 1990), which could be critical in cases where outcomes are used to drive financial decisions made by business professionals and where inappropriate actions taken on inaccurate results could be devastating (Canhoto, 2015).

\section{TECHNOLOGICAL AND THEORETICAL BACKGROUND}

Many AI based approaches exist to address this issue. Some low-level frameworks, like TensorFlow, Theano, and SKLearn allow data scientists to train neural networks to identify negative content (Bahrampour, 2016) (Wu, 2018). This process generally involves the standard steps in any natural language processing effort including tokenizing words, which is the process of splitting text into word-like pieces for processing, removing stop words which are the most common functional words in a language, creating feature vectors from tokens which can be used as input data for model training, etc. In addition to these low-level approaches, which would only be available to data scientists skilled in the art, many cloud computing vendors provide high level services to enable a broader range of users to perform sentiment analysis (Liu, 2018). This project performs experiments with the two services available on the Google Cloud Platform (GCP) for performing sentiment analysis: Natural Language API and AutoML Natural Language. Comparisons are made between the two services using the dataset described in the next section. 


\section{DATA}

This section covers all of the key topics that relate to the data used in the project including how the data was labeled and how the data was cleaned to address issues.

\section{Background of Data}

This project takes inspiration from two related Kaggle competitions on sentiment analysis. The first competition, called “Toxic Comment Classification Challenge”, provides data from a large number of Wikipedia comments which were labeled by human raters for toxic behavior. The second competition, called "Jigsaw Unintended Bias in Toxicity Classification", leverages data collected from the now defunct "Civil Comments" platform, which was a full-featured commenting plugin for independent news sites. Labels for the data were generated by the Civil Comments users who were motivated to provide ratings of the comments by the platform's peer-review submission process. Kaggle performed the original sourcing of the data to support both competitions and in turn makes the data publicly available for research purposes. The data used in this project was retrieved directly from the Kaggle website.

\section{Data Preprocessing}

The datasets retrieved from the Kaggle competitions referenced above provide over 1.5 million samples of labeled comments for sentiment analysis. This section outlines the preprocessing steps taken to prepare the data for analysis on the GCP.

Data cleansing of the original data:

a. End-of-line characters were embedded in many of the comment strings, which caused parsing errors with the file. This issue was addressed using a series of UNIX command line tools to temporarily mark the true end-of-line location, then removing all end-of-line and carriage return characters from the entire file, and then finally replacing the temporary marker with the true end-of line character.

b. All embedded double quotes were removed from comments to avoid parsing errors with the eventual .csv file that would be used for the AutoML model training.

c. All non-English characters were removed from the comment strings. Neither of the GCP sentiment analysis services support non-English charter sets.

Addressing unbalanced data:

a. The original data contained the following counts of target classes:

Total count of positive sentiment messages: 1,675,189

Total count of negative sentiment messages: 104,796

b. To support the AutoML Natural Language model training, it was required to have a similar number of samples of messages in both classes (positive and negative message sentiments).

c. Under-sampling was performed on both classes of messages to produce the following breakdown of messages:

Total positive sample count: 50,000

Total negative sample count: $\mathbf{5 0 , 0 0 0}$

NOTE: At the time of this writing, GCP had a limit of 100,000 records for performing AutoML Natural Language model training.

Addressing duplicated data:

Unfortunately, duplicate comments were found in the data. A Python routine was developed to eliminate all duplicate message strings to protect the integrity of the separate data sets (train/validation/test) which will be discussed below.

Interpolating toxicity scores:

a. The original data contains target scores ranging from 0 to 1 , and were fractional values representing the percent of human raters who believed toxic sentiment applied to the given comment. A target $>=0.5$ is considered to be in the positive class (toxic) 
b. The Natural Language API uses sentiment ranges between -1.0 (toxic) and 1.0 (positive) with 0.0 representing relatively neutral text.

c. The AutoML Natural Language offering used a flexible scale starting from 0 (toxic) up to a maximum sentiment level (positive or non-toxic sentiment) defined by the user depending on how granular the assessment is required to be.

d. For this project, all target labels in the data were interpolated onto a scale of 0 (toxic) to 1 (non-toxic) in accordance with the most basic AutoML scale. This was accomplished using the numpy.interp() function.

Splitting the data:

The GCP AutoML offering provides an option to label each record as "TRAIN", "VALIDATION", or "TEST" when the user wants to assert control over how each record would be used. This option was ideal for this project because the intention was to test both GCP sentiment analysis offerings with the same set of records. With this in mind, the 100,000 records described above were further split using an 80/10/10 ratio as shown below:

Train record count: 80,000 [positive:40,000 and negative 40,000 ]

Validation record count: 10,000 [positive:5,000 and negative 5,000]

Test record count: 10,000 [positive:5,000 and negative 5,000]

\section{EXPERIMENT 1: NATURAL LANGUAGE API}

This section describes the process taken to execute the Natural Language API and the resulting quality analysis of the results.

\section{Executing Natural Language API}

The Natural Language API prebuilt models do not require training and validation data. Only the records tagged "TEST", as described above in the "Splitting the data" section, were used in this experiment.

The GCP provides multiple APIs for invoking the natural language service for sentiment analysis, and Python was selected for use in this project.

High-level steps required for execution:

a. Logon to the GCP console to:

i. $\quad$ Create a project

ii. Enable the Google Natural Language API

iii. $\quad$ Create a service account

iv. Download a private key as a JSON file and set the environment variable GOOGLE_APPLICATION_CREDENTIALS to the file

b. Install the google-cloud-language client library into a virtual Python environment.

c. Execute sentiment analysis using the Python API. The salient portions of the API are highlighted below:

\# Wrap the comment string in a Document object

document $=$ types.Document $($ content=comment_text, type=enums.Document.Type.PLAIN_TEXT)

\# Detect the sentiment of the Document

sentiment $=$ client. analyze_sentiment(document=document).document_sentiment

\section{NATURAL LANGUAGE RESULTS}

The results of the Python API invocation described above were compared to the labeled test data to determine the overall quality of the sentiment analysis performed by the GCP Natural Language API. The results are presented in this section.

The confusion matrix displayed in Figure 1 below indicates that the Natural Language API has a particularly tough 
time with false positives, i.e. non-toxic comments (sentiment 1 ) were incorrectly classified as being toxic (sentiment 0 ). Almost $40 \%$ of the predication fell into this category. In addition, the overall accuracy was only $57 \%$.

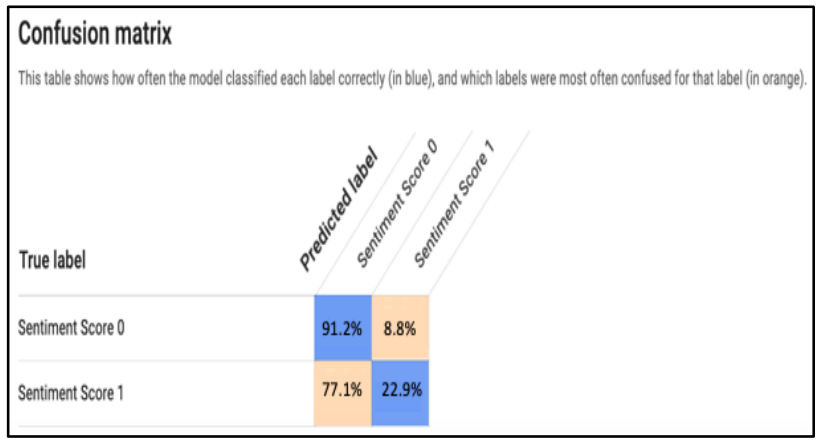

Figure 1. ROC Curve for Negative Sentiment

The following table outlines other key metrics describing the quality of the sentiment analysis. Looking at the numbers, we can see that the precision score for toxic messages is low, which explains the large number of false positives in Figure 1. In addition, the model very rarely predicts a message to be non-toxic, as shown by the low recall score.

\begin{tabular}{|c|c|c|c|c|}
\hline tabled & Predison & Rerall & FSore & Support \\
\hline Toxic & 0.54 & 0.91 & 0.68 & 5000 \\
\hline Non Toxic & 0.72 & 0.23 & 0.35 & 5000 \\
\hline
\end{tabular}

Figure 2. Natural Language Quality Metrics

One final graphic describing the quality of the model is shown below. The low area under the curve (AUC), shows the low quality of the predictions of the model.

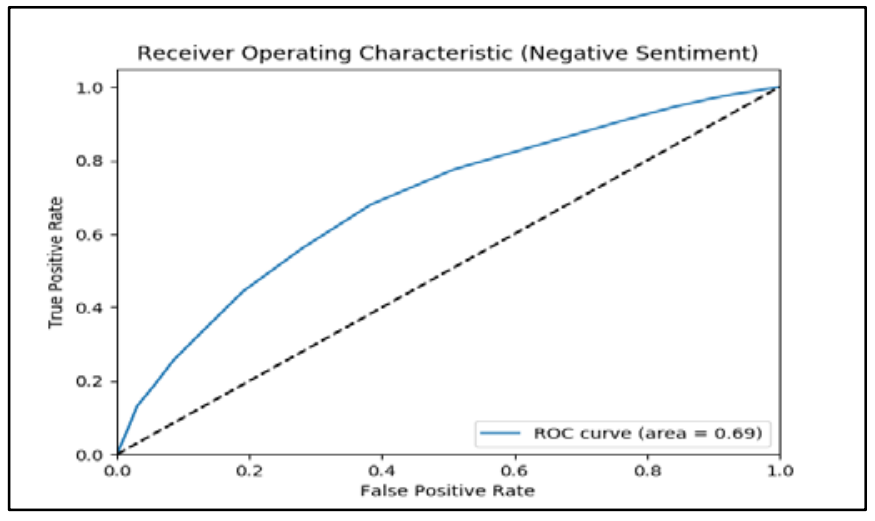

Figure 3. ROC Curve for Negative Sentiment

\section{EXPERIMENT 2: AUTOML NATURAL LANGUAGE}

The goal of the AutoML Natural Language offering is to enable users to train custom models in cases where the Natural Language API does not perform well. This can happen with challenging datasets, especially those 
involving domain specific terms and phrases. This section describes the process taken to train the AutoML Natural Language custom model and the quality of the results.

\section{Training an AUTOML Custom Model}

When training a custom AutoML model, labeled data is required. In this experiment, we use all of the prepared data previously tagged as "TRAIN", "VALIDATION", and "TEST". The AutoML service will automatically train a model using the training and validation data. After the training is complete, the service uses the testing data to determine the true quality of the sentiment analysis and makes various quality metrics available on the GCP console.

The GCP provides multiple APIs for training a custom AutoML model. The standard web user interface, which is a part of the GCP console was use for this experiment.

High-level steps required for execution:

a. Prepare an input file in the following format:

TRAIN,"...message text...",0

VALIDATION,"...message text ...",0

TEST,"... message text...",1

NOTE: Each record is labeled as "TRAIN", "VALIDATION", or "TEST" to indicate how it should be used.

b. Logon to the GCP console to:

i. $\quad$ Create a dataset

ii. Import the data from a local .csv file. Records are validated in this step during the upload. Common errors include:

- Non-English characters found in the comment

- Duplicate comments found

- Unsupported labels in column 1. Only "TRAIN", "VALIDATION", or "TEST" are expected.

- This process took about two hours of runtime to complete. An email was sent when the upload was complete.

iii. Initiate the training, which took approximately 5 hours of runtime for this experiment. An email was sent when the training was complete.

\section{AUTOML CUSTOM MODEL RESULTS}

The results of the sentiment analysis using the trained custom model were generated by the AutoML process using the records labeled as "TRAIN" in the dataset. The results are presented in this section.

\section{Confusion Matrix Comparison}

The confusion matrix displayed in Figure 4 highlights the improvements that were made by the custom AutoML model. Recall that the Natural Language API model had a particularly tough time with false positives. Almost $40 \%$ of the original predications were false positives. Now we can see that roughly $6 \%$ of the predictions are false positives, and the overall accuracy has improved from $57 \%$ to $90 \%$. The two models appear similar in their ability to correctly identify negative sentiment (the true positive rates for sentiment score 0 are similar), but the AutoML model is much better at correctly identifying positive sentiment (sentiment score 1) since the false positive issue has been resolved. 


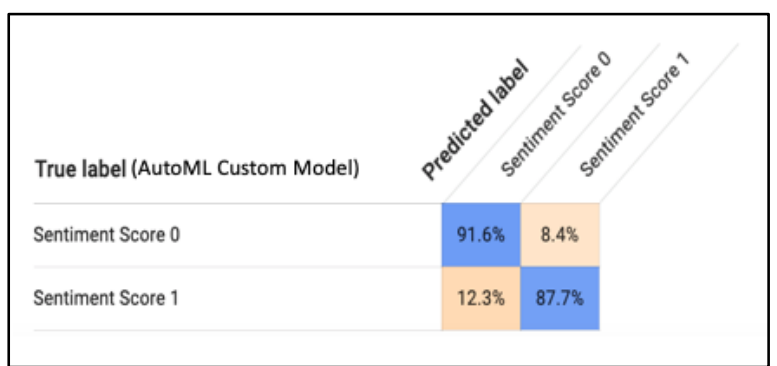

Figure 4. Confusion Matrix Comparison

\section{Quality Metrics Comparisons}

The following table outlines the improvements made with the other key metrics describing the quality of the sentiment analysis. When we looked at the improved confusion matrix, we noticed that the two models were similar in their ability to correctly identify negative sentiment, but here we see that AutoML is more precise in this regard. Also, we see a dramatic improvement in recall for non-toxic message. The new model is much more likely to predict the nontoxic classes when appropriate.

\begin{tabular}{|c|c|c|c|c|}
\hline \multicolumn{5}{|c|}{ AutoML Custom Model } \\
\hline Label & Precision & Recall & F-Score & Support \\
\hline Toxic & 0.88 & 0.92 & 0.90 & 5000 \\
\hline Non Toxic & 0.91 & 0.88 & 0.89 & 5000 \\
\hline
\end{tabular}

Figure 5. Quality Metrics Comparisons

\section{CONCLUSION AND RETROSPECTIVE}

The improvements highlighted in the quality metrics above clearly demonstrate the benefits of building a custom model using the AutoML Natural Language service offered on the GCP platform. In addition to its ease of use and convenient reporting of the model's quality metrics, AutoML also highlights some specific examples that were misclassified to assist with the potential retraining of the model, and to provide insights on the type of messages the model struggled to classify. Here are some samples of misclassified messages from the model that was built for this project: 


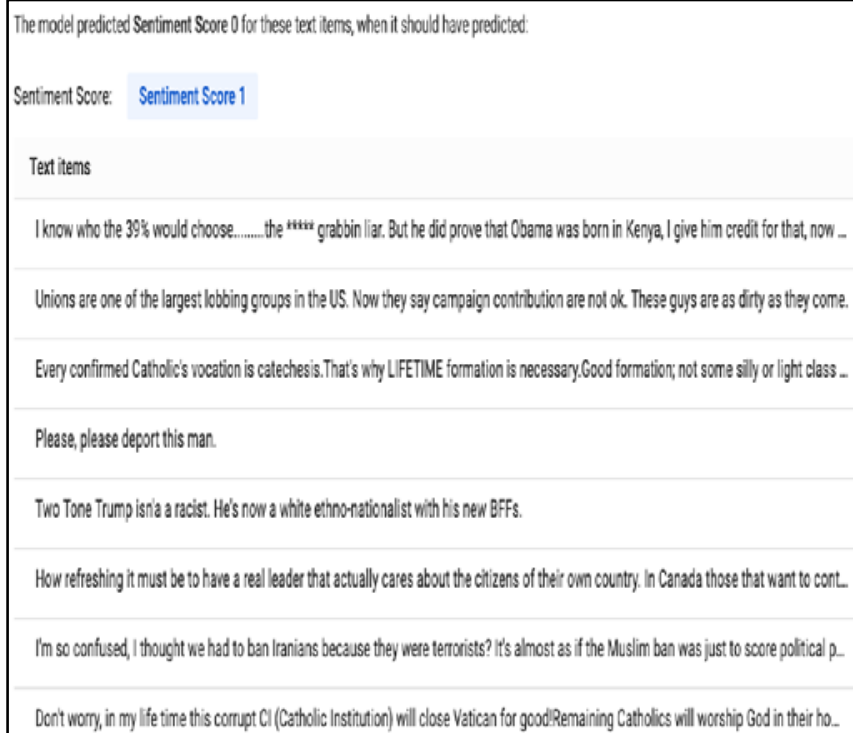

Figure 6. False positives from the perspective of the toxic class

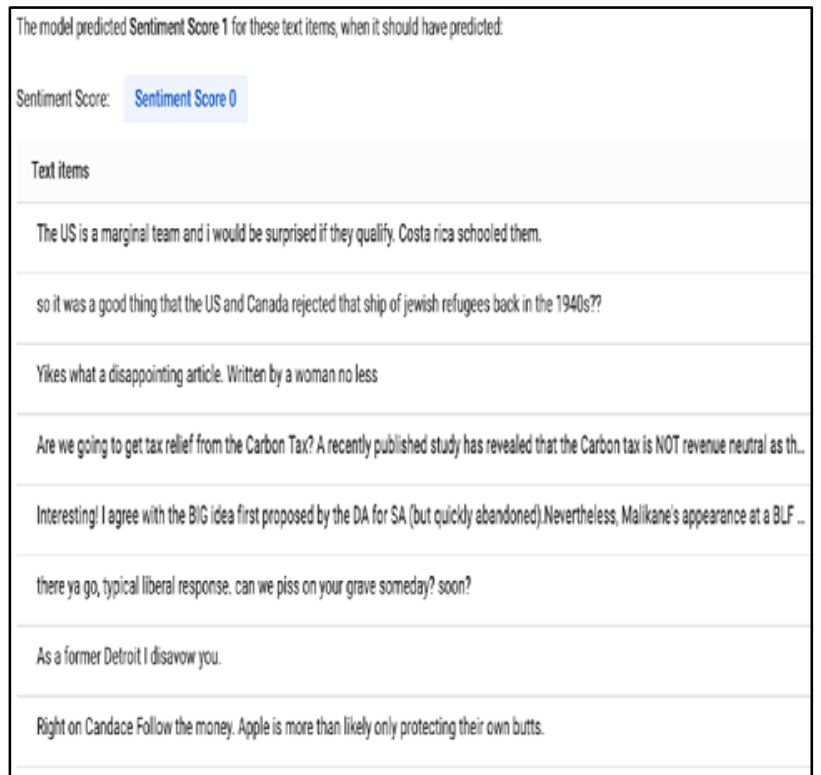

Figure 7. False positives from the perspective of the non-toxic class

Figure 6 above lists a few examples of messages that were originally labeled by the human raters assigned to assess our original dataset as being non-toxic (score 1), but it is easy to browse this list and find a few that some other people might have labeled toxic (score 0). Figure 7 lists a few messages that were labeled by our human raters as toxic, but even here, there are a few cases that can be made for a different label. This shows the subjective nature of sentiment analysis and drives home the point that not even humans can reach a consensus on every message. This makes the model's job much more difficult, and cautions data scientists to be careful as they interpret the results of any sentiment analysis. Objective truth exists, but it should not be carelessly taken for granted when we receive 


\section{Issues in Information Systems}

Volume 21, Issue 2, pp. 221-228, 2020

labeled data to be used for AI model training. Ultimately all labeled sentiment analysis data is subjective, and the model is being trained to predict sentiment based on the subjective views of the group of humans responsible for labeling the data. Thus, the model will be subject to the inherent bias of the group of people chosen to assess the message content.

\section{POSSIBLE EXTENSIONS TO THE WORK}

Based on the discussion of subjectivity covered in the previous section, one interesting extension to this work could be to present the same set of messages to different groups of people across the country to get multiple sets of labels describing the toxicity of the same set of messages. For example, we could produce labeled data from more conservative parts of the country, and a different set of labels from liberal parts of the country. The group of raters could also be broken down in other ways, like by race, age, religious background, nationality, etc. We could build multiple models using the same techniques on various sets of labeled data to study the differences in sentiment, and potential bias, amongst various groups. In this approach, the data scientist would keep the model's architecture and hyperparameters consistent, and vary the labels from model to model, then make comparisons with the results.

A more technical extension of this project might include a lower level TensorFlow model on the GCP platform where the data scientist would have more control over the model's architecture. For this extension to the work, the modeler would work with a single set of labels, and experiment with various model configurations and vary the hyperparameters using Tensor flow in an attempt to improve the model's performance above what was achieved with the AutoML service offering.

\section{REFERENCES}

Bahrampour et al, (2016). “Comparative Study of Deep Learning Software Frameworks”, arXiv.org, Cornell University Library, United States, Ithaca

Bandeli,Kiran Kumar and Agarwal,Nitin (2018). "Analyzing the role of media orchestration in conducting disinformation campaigns on blogs", Computational and Mathematical Organization Theory, Springer Science+Business Media, LLC, 1-27.

Brown et al, (1990). "Computers and QDA - can they help it? A report on a qualitative data analysis programme”, The Sociological Review, Wiley Blackwell, Vol.38(1), 134-150.

Buzzetto-More,Nicole, Johnson,Robert and Elobaid,Muna (2015). "Communicating and sharing in the semantic web: an examination of social media risks, consequences, and attitudinal awareness.”, Interdisciplinary Journal of e-Skills and Lifelong Learning (Vol. 11), Informing Science Institute, 47 (20).

Canhotol,Isabel and Padmanabhan, Yuvraj (2015). “We (don't) know how you feel' - a comparative study of automated vs. manual analysis of social media conversations”, Journal of Marketing Management, Taylor \& Francis Group, UK, Vol. 31 Nos 9/10, 1141-1157.

Gaspar,Rui, Pedro,Claudia, Panagiotopoulos,Panos, and Seibt,Beate (2015). "Beyond positive or negative: Qualitative sentiment analysis of social media reactions to unexpected stressful events", Computers in Human Behavior, Elsevier, 179-191.

Islam,Md Rakibul and Zibran,Minhaz F. (2017). "Leveraging Automated Sentiment Analysis in Software Engineering”, IEEE/ACM 14th International Conference on Mining Software Repositories (MSR), 203-214.

Liu, Bin (2018). “A Very Brief and Critical Discussion on AutoML”, Nanjing University of Posts and Telecommunications, Nanjing, 210023 China

Wu,Yanzhao, Cao,Wenqi, Sahin,Semih, and Liu,Ling (2018). "Experimental Characterizations and Analysis of Deep Learning Frameworks”, BMC Bioinformatics Vol.19 (Suppl 8)., IEEE 